\title{
A randomised study on the effectiveness of therapist-led $v$. self-help parental intervention for treating childhood obesity
}

\author{
Hilde Tang Hystad ${ }^{1}$, Silje Steinsbekk ${ }^{2,3}$, Rønnaug $\varnothing$ degård $^{4,5}$, Lars Wichstrøm² ${ }^{2}$ and \\ Oddrun Anita Gudbrandsen ${ }^{1 *}$ \\ ${ }^{1}$ Institute of Medicine, Haukeland University Hospital, University of Bergen, N-5021 Bergen, Norway \\ ${ }^{2}$ Department of Psychology, Norwegian University of Science and Technology, Dragvoll, 7491 Trondheim, Norway \\ ${ }^{3}$ Department of Child-and Adolescent Psychiatry, St Olav University Hospital, Olav Kyrres Gate 17, 7030 Trondheim, \\ Norway \\ ${ }^{4}$ Department of Pediatrics, St Olav University Hospital, Olav Kyrres Gate 17, 7006 Trondheim, Norway \\ ${ }^{5}$ Department of Laboratory Medicine, Children's and Women's Health, 7433 Trondheim, Norway \\ (Submitted 22 August 2012 - Final revision received 26 November 2012 - Accepted 21 December 2012 - First published online 7 February 2013)
}

\begin{abstract}
A strategy is needed on how to treat the growing number of obese children with the limited resources available. We compared the longterm (24 months) effectiveness of therapist-led groups (TLG) $v$. self-help groups (SHG) for parents on changes in children's adiposity and dietary intake. The study included ninety-nine children (forty-eight girls) who were referred to obesity treatment (7-12 years, BMI $z$-scores $\geq 2$, attendance of at least one parent). Parents (ninety-one mothers, fifty-four fathers) were randomised to TLG aimed at increasing parents' competence to accomplish lifestyle changes ( $n$ 47), or SHG ( $n$ 52), both with fifteen sessions. All children participated in children's groups, and all families attended individual counselling by a clinical dietitian and physiotherapist. Percentage of body fat (BF) was measured by dual-energy X-ray absorptiometry, BMI $z$-score was calculated by international reference values and dietary intake was calculated from $4 \mathrm{~d}$ estimated food records at baseline and after 6 and 24 months. No significant between-group differences were detected in the children's changes in adiposity or dietary intake after 6 and 24 months. BF, BMI $z$-scores and energy intake were significantly decreased after 6 months $(P<0.05)$ in both intervention groups, and this persisted throughout 24 months without compromising the diet macronutrient composition. In conclusion, the TLG and SHG intervention groups appear to be equally effective in improving long-term adiposity and dietary intake in obese children. Further research should be performed to clarify whether the SHG should be preferred to parental group treatment for similar children with obesity.
\end{abstract}

Key words: Obesity treatment: Obese children: Dietary intake

Interventions targeting a combination of increased physical activity, reduced sedentary behaviour and promotion of healthy eating have been shown to be most promising in the treatment of childhood obesity ${ }^{(1)}$. Increased treatment effect is seen in programmes where the parents are targeted as the main agents of change ${ }^{(2)}$, enhancing parental leadership, parenting skills and taking advantage of the parents' ability to modify the shared family environment ${ }^{(3)}$. However, the most effective way to involve parents in these interventions remains unclear and warrants further investigation ${ }^{(4,5)}$.

Many studies on multi-component childhood obesity treatment are too short in duration to see a significant reduction in adiposity, but still focus solely on adiposity outcomes and may therefore underestimate the interventions' effectiveness $^{(2)}$. Measuring changes in health-related behaviours, such as dietary intake, may be an alternative indicator of treatment effect ${ }^{(2)}$ when the duration of the study is too short to see the effects on adiposity. A systematic review of randomised trials found that interventions including dietary modification are effective in reducing the adiposity of obese children, but details of the dietary intervention or participants' dietary intake are rarely described ${ }^{(6)}$.

The present study is a family-based child obesity trial with different parental interventions: therapist-led groups (TLG) and self-help groups (SHG). The objective of the present study was to compare the long-term ( 24 months) effectiveness of these interventions by assessing changes in adiposity and dietary intake, by achieving changes in lifestyle based on the

Abbreviations: BF, body fat; E\%, percentage of energy intake; SHG, self-help group; TLG, therapist-led group.

*Corresponding author: O. A. Gudbrandsen, fax +47559758 90, email oddrun.gudbrandsen@med.uib.no 
families' situation at baseline. This is thus one of few trials existing with assessment beyond 1 year ${ }^{(7)}$ and the first to compare the long-term changes in adiposity and dietary intake accompanying different ways to involve parents in familybased obesity treatment of children. Details of the dietary intervention and participants' dietary intake are described.

\section{Methods}

\section{Participants, study setting and ethics}

Children who were referred by their general practitioner to outpatient obesity treatment at St Olav University Hospital, Trondheim, Norway, in 2005-8, were assessed for eligibility. The inclusion criteria were as follows: age 7-12 years; BMI $z$-scores $\geq 2$; participation of at least one parent; the ability to participate in a group setting. Families were excluded if the obese child was mentally retarded, if there was an organic cause of obesity or if the child used medication that may interfere with growth or weight control. No data on puberty stage of the participating children were recorded.

The treatment was conducted in an outpatient hospital setting. Only accredited psychologists, paediatricians, clinical dietitians and physiotherapists with experience of treating children with obesity were permitted to manage patients in the trial. The present study was conducted according to the guidelines laid down in the Declaration of Helsinki, and all procedures involving patients were approved by the Regional Ethical Committee for Medical Research. Written informed consent was obtained from all parents. All data were analysed anonymously. The registration number of the present trial is nct00842777.

\section{Interventions}

Reduced child adiposity was targeted through gradual changes that the families could manage to maintain over time, based on international and Norwegian recommendations ${ }^{(8,9)}$. The focus of both the TLG and SHG interventions was to establish regular mealtimes, increase the intake of fruits, vegetables and other high-fibre food, reduce the intake of added sugar and fat, conduct at least $1 \mathrm{~h}$ of moderate physical activity per $\mathrm{d}$ and reduce sedentary behaviour gradually, towards a maximum of $2 \mathrm{~h}$ per $\mathrm{d}$.

The main focus of the TLG sessions was to enhance the parents' competence to accomplish the targeted lifestyle changes. A detailed treatment manual was devised. A total of ten sessions were conducted with the following topics: expectancies and goal setting; communication about obesity, diet and physical activity; daily physical activity; everyday dietary habits; mastery and motivation; guidance and setting boundaries; the role of siblings and the social network; parent's history of diet and physical activity; self-concept and body image; vacations and birthday parties. In brief, each group session was led by two therapists, and each session included the following: a presentation of the topic of the session followed by a group discussion; a discussion of the homework assignment for the present session; in some sessions also a role play on the topic of the session. A series of written material, such as 'fridge notes', home activity sheets and goal attainment sheets, was developed.

The SHG were based on the principle of mutual help, derived from the participants' own experiences and knowledge. A health professional attended the two first and the last meeting to organise the group and facilitate group rules, but did not offer any education or guidance regarding how to reduce adiposity.

Stratified by the age, sex and BMI of their child, parents were randomly allocated to the TLG or SHG intervention (1:1 ratio) using a computer-generated list of random numbers. Both the TLG and SHG consisted of parents from four to six families.

All children, regardless of their parents' group affiliation, participated in age-matched groups of six to twelve children led by a clinical dietitian and a physiotherapist. The aim was for the children to gain positive experiences related to physical activity and healthy eating, and the psychosocial consequences of being obese were addressed in a session led by a psychologist. All families attended five individual counselling sessions with a clinical dietitian and a physiotherapist to discuss the family's progress and to define new goals.

The design of the study was based on the findings from pilot studies from 2003 to 2005, suggesting that it was preferable to have an intensive phase of 6 months at the beginning of the intervention period followed by a longer and less intensive phase of 18 months (S Steinsbekk and R Ødegård, unpublished results). The TLG, SHG and children's groups met simultaneously every second week for ten sessions during the first 6 months. During this 6-month period, each family also met monthly for individual counselling. Over the remaining 18 months of the 24-month intervention, the groups met five times at the hospital, and four individual family counselling sessions were conducted. Each of the fifteen group sessions lasted $2 \mathrm{~h}$, while each of the ten individual family counselling sessions lasted 30 min. The study was completed in February 2010.

\section{Outcome measurements}

The primary study outcomes of the present study were changes in the percentage of body fat (BF), BMI $z$-scores and dietary intake. Data were collected in the hospital setting by members of the treatment staff at baseline (before randomisation), and after 6 and 24 months of treatment. Apart from the health professionals performing the dual-energy X-ray absorptiometry, it was not possible to keep assessors blinded to treatment condition. A standard protocol was used to facilitate the objective and reliable measurement of height and weight.

During anthropometric assessments, children wore light clothing and no shoes. Weight was obtained by a digital scale (Seca 930; Vogel\&Halke) and height was measured by a stadiometer (Hyssna Limfog AB). BMI was calculated in $\mathrm{kg} / \mathrm{m}^{2}$, and BMI $z$-score was computed according to international reference values ${ }^{(10)}$. Dual-energy X-ray absorptiometry (Hologic QDR Discovery) was used to estimate BF.

A $4 \mathrm{~d}$ food record (three consecutive weekdays and one weekend day) was used to estimate the children's dietary intake. Children and parents were instructed to register 
everything the children ate and drank during the $4 \mathrm{~d}$ period. To improve the participants' accuracy in reporting portion sizes, a booklet ${ }^{(11)}$ with a photograph series of thirteen food items of known portion weights was distributed to the families for comparison instead of weighing all food. A clinical dietitian reviewed the food record together with the family at the forthcoming individual consultation. Daily energy and macronutrient intake was calculated from the reported food intakes using a Web-based dietary analysis program based on the Norwegian Food Composition Table 2006 (Mat på Data 5.1; Norwegian Food Safety Authority, the Directorate for Health and the Department of Nutrition at the University of Oslo), and was compared with Norwegian dietary recommendations ${ }^{(9)}$ : protein $(10-20 \%$ of energy intake (E\%)); fat (25-35 E\%); SFA ( $\leq 10 \mathrm{E} \%) ;$ MUFA (10-15 E\%); PUFA (5-10 E\%); carbohydrates (50-60 E\%); added sugar ( $\leq 10 \mathrm{E} \%)$; dietary

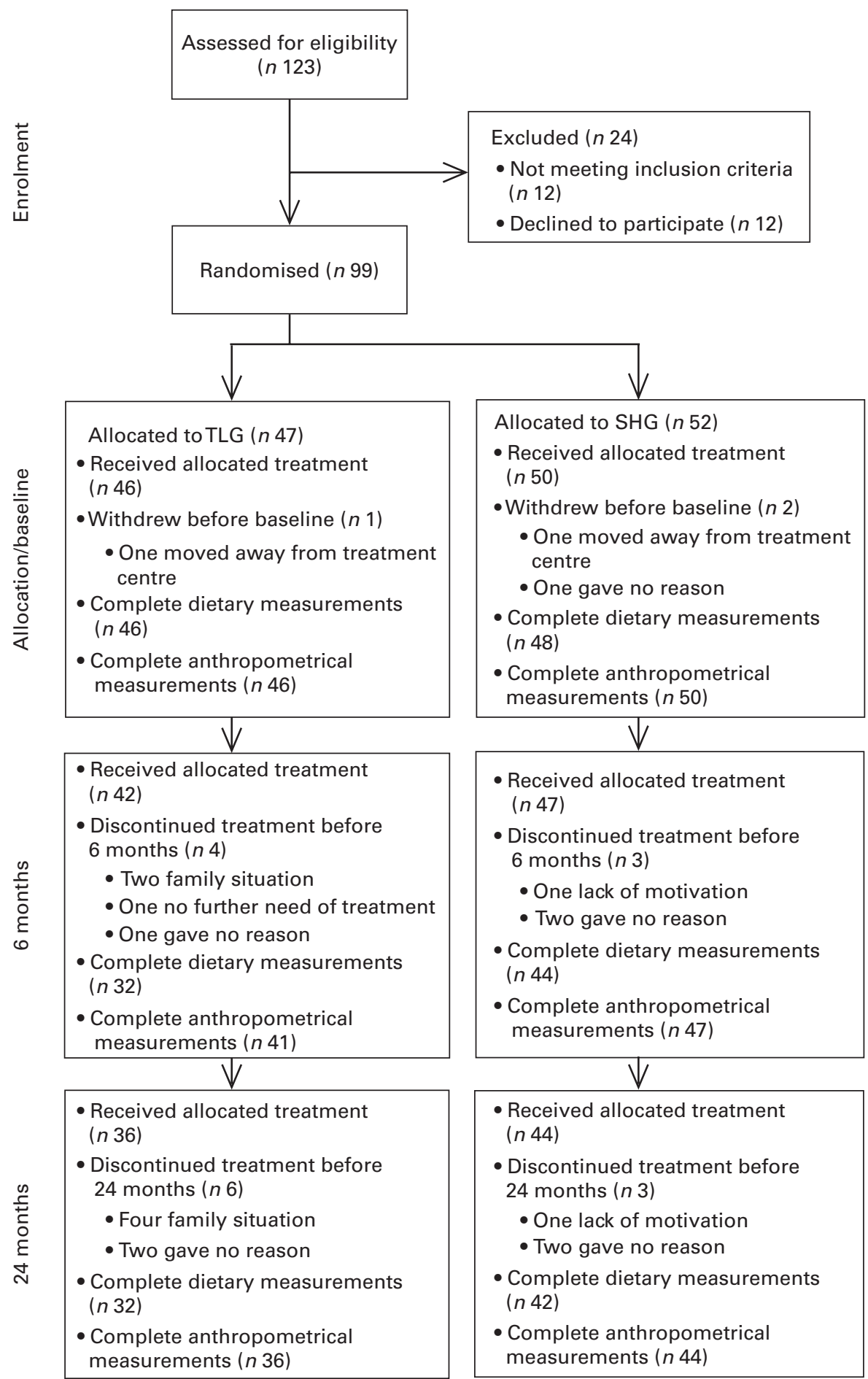

Fig. 1. Flow diagram of the participants' course throughout the study, randomly allocated to the parallel therapist-led group (TLG) or self-help group (SHG). 
fibre $(2-3 \mathrm{~g} / \mathrm{MJ})$. Intake of macronutrients was expressed both as grams and as $\mathrm{E} \%$ and fibre as nutrient density $(\mathrm{g} / \mathrm{MJ})$, because children aged 7-12 years have different requirements for amount of food, whereas the same macronutrient composition is recommended for this age group ${ }^{(9)}$. Energy intake was reported per $\mathrm{kg}$ of body weight and as a ratio of energy intake:estimated BMR (energy intake:BMR). BMR was estimated from weight and height using age- and sex-dependent Schofield equations $^{(12)}$.

\section{Statistical methods}

The distribution of the data was found to be normally distributed, and comparisons of baseline study characteristics between the groups, and between-group differences for the mean change in outcomes from baseline to 6 and 24 months were analysed with independent-samples $t$ tests, using PASW 19.0 software (IBM Corporation). Within-group parameter changes were analysed using a paired $t$ test. Statistical significance was set at $P<0 \cdot 05$. To correct for multiple comparisons to control the expected proportion of incorrectly rejected null hypotheses, the calculated $P$ values were corrected for multiple testing by a false discovery rate using the Benjamini-Hochberg method ${ }^{(13)}$.

\section{Results}

\section{Participants}

The flow of participants throughout the trial and the number of participants in each group from whom anthropometrical and dietary data were obtained at baseline, 6 and 24 months are described in Fig. 1. Of the 123 families assessed for eligibility, ninety-nine (80.5\%) consented (forty-eight girls, fifty-one boys) and were randomly assigned to the TLG
( $n$ 47) or SHG ( $n$ 52) intervention. The retention rate was $90 \%$ after 6 months and $81 \%$ after 24 months, and there were no differences in retention rates between the groups.

Not all children handed in their food record forms at all visits, and it was of interest to see whether there were any differences between the children who handed in their forms and those who did not. As shown in Fig. 1, most children in both intervention groups did hand in their food record forms at baseline (TLG, $100 \%$; SHG, 96\%), and after 6 months (TLG, 76\%; SHG, 94\%) and 24 months (TLG, $89 \%$; SHG, 95\%). Regarding age, sex, BF, BMI $z$-score and energy intake at baseline, there were no differences between participants who handed in food record forms at 6 and 24 months, compared with those who did not (data not shown).

At baseline, the mean $\mathrm{E} \%$ from SFA in both intervention groups was above the recommended amount ( $\leq 10 \mathrm{E} \%$ ), while the TLG's mean E\% from added sugar was above the recommended amount $(\leq 10 \mathrm{E} \%$ ) and the SHG's mean $\mathrm{E} \%$ from carbohydrates was below the recommended amount (50-60 E\%). The mean $\mathrm{E} \%$ of the remaining variables was in accordance with Norwegian recommendations ${ }^{(9)}$.

There were no statistically significant differences between the two groups at baseline, regarding child and parent anthropometry, child age, sex, energy intake and BMR (Table 1). All but two of the participating children were Caucasian; one child was of African origin and another was of Latin American origin.

\section{Changes during the treatment}

Except for the change in $\mathrm{E} \%$ from added sugar from baseline to 6 months (TLG, $-2 \cdot 8$ (SD 4.4 ); SHG, 0.9 (SD 7.2); $P<0 \cdot 05$ ), there were no significant between-group differences for the change in BF, BMI $z$-scores or dietary intake from baseline to 6 and 24 months (data not shown).

Table 1. Baseline characteristics of obese children ( $n$ 83) and their parents, participating in a randomised controlled trial by treatment group and total group*

(Mean values and standard deviations)

\begin{tabular}{|c|c|c|c|c|c|c|}
\hline & \multicolumn{2}{|c|}{$\begin{array}{l}\text { Therapist-led group } \\
\qquad(n 39)\end{array}$} & \multicolumn{2}{|c|}{$\begin{array}{l}\text { Self-help group } \\
\qquad(n 44)\end{array}$} & \multicolumn{2}{|c|}{$\begin{array}{l}\text { Total } \\
(n 83)\end{array}$} \\
\hline & Mean & SD & Mean & SD & Mean & SD \\
\hline Age (years) & $9 \cdot 9$ & 1.5 & $10 \cdot 5$ & 1.9 & $10 \cdot 2$ & $1 \cdot 7$ \\
\hline \multicolumn{7}{|l|}{$\operatorname{Sex}(n)$} \\
\hline Male & \multicolumn{2}{|c|}{23} & \multicolumn{2}{|c|}{27} & \multicolumn{2}{|c|}{39} \\
\hline Female & \multicolumn{2}{|c|}{24} & \multicolumn{2}{|c|}{25} & \multicolumn{2}{|c|}{44} \\
\hline Weight (kg) & $59 \cdot 8$ & $15 \cdot 6$ & $64 \cdot 6$ & $15 \cdot 9$ & $62 \cdot 3$ & $15 \cdot 9$ \\
\hline Height (m) & 1.44 & $0 \cdot 10$ & 1.49 & 0.12 & 1.46 & $0 \cdot 11$ \\
\hline $\mathrm{BMI}\left(\mathrm{kg} / \mathrm{m}^{2}\right)$ & $28 \cdot 4$ & 4.5 & $28 \cdot 7$ & 3.5 & $28 \cdot 6$ & $4 \cdot 0$ \\
\hline BMI z-score† & 3.00 & 0.51 & 3.00 & 0.36 & 3.00 & 0.43 \\
\hline Body fat $(\%) \ddagger$ & $40 \cdot 4$ & $3 \cdot 8$ & $40 \cdot 6$ & $4 \cdot 0$ & $40 \cdot 5$ & $3 \cdot 9$ \\
\hline Waist circumference $(\mathrm{cm})$ & $93 \cdot 5$ & $12 \cdot 7$ & $96 \cdot 0$ & $10 \cdot 0$ & $94 \cdot 8$ & $11 \cdot 3$ \\
\hline EI§ (kJ/d) & 7317 & 1627 & 7499 & 1573 & 7414 & 1591 \\
\hline BMR\| (kJ) & 6670 & 1162 & 7051 & 1311 & 6870 & 1251 \\
\hline Parent BMI $\left(\mathrm{kg} / \mathrm{m}^{2}\right)$ & $32 \cdot 0$ & 7.4 & 31.7 & 6.7 & $31 \cdot 8$ & $7 \cdot 0$ \\
\hline
\end{tabular}

El, energy intake.

${ }^{*}$ Mean values were not significantly different between the intervention groups $(P>0.05)$.

†BMI $z$-score was calculated according to Cole et al. ${ }^{(10)}$.

$\ddagger$ Body fat was measured by dual-energy $X$-ray absorptiometry.

$\S$ El was estimated from $4 \mathrm{~d}$ food records.

|l BMR was calculated with Schofield equations ${ }^{(12)}$. 
Table 2. Measures of adiposity and dietary intake at baseline, 6 and 24 months of the study participants by treatment group, and changes from baseline to 6 and 24 months§ $§$ (Mean values and standard deviations)

\begin{tabular}{|c|c|c|c|c|c|c|c|c|c|c|c|c|}
\hline & \multicolumn{6}{|c|}{ TLG } & \multicolumn{6}{|c|}{ SHG } \\
\hline & \multicolumn{2}{|c|}{ Baseline } & \multicolumn{2}{|c|}{6 months } & \multicolumn{2}{|c|}{2 years } & \multicolumn{2}{|c|}{ Baseline } & \multicolumn{2}{|c|}{6 months } & \multicolumn{2}{|c|}{2 years } \\
\hline & Mean & SD & Mean & SD & Mean & SD & Mean & SD & Mean & SD & Mean & SD \\
\hline Body fat $(\%)$ & 40.4 & 3.8 & $35 \cdot 7^{\star \star \star} \dagger$ & 5.6 & $35 \cdot 6^{\star \star} \dagger$ & $6 \cdot 3$ & $40 \cdot 6$ & 4.0 & $36 \cdot 2^{\star \star \star} \dagger$ & $5 \cdot 6$ & $35 \cdot 6^{\star \star \star} \dagger$ & 6.4 \\
\hline BMI $z$-score $\neq \neq$ & 3.00 & 0.51 & $2 \cdot 78^{\star \star \star} \dagger$ & 0.56 & $2 \cdot 82^{\star \star} \dagger$ & 0.59 & 3.00 & 0.36 & $2 \cdot 81^{\star \star *} \dagger$ & 0.44 & $2 \cdot 83^{\star \star} \dagger$ & 0.51 \\
\hline EI§§ (kJ) & 7317 & 1627 & $6454^{\star *} \dagger$ & 1216 & $6694^{\star \star} \dagger$ & 1107 & 7499 & 1573 & $6693^{* *} \dagger$ & 1573 & $6701^{* * *} t$ & 1553 \\
\hline $\mathrm{El} / \mathrm{kg}$ & 128 & 33.7 & $108 \cdot 7^{\star \star} \dagger$ & 33.0 & $95 \cdot 1^{\star \star \star} \dagger$ & $25 \cdot 2$ & $121 \cdot 3$ & $37 \cdot 1$ & $104 \cdot 0^{\star \star \star} \dagger$ & 33.3 & $89 \cdot 0^{\star \star \star} \dagger$ & $24 \cdot 1$ \\
\hline El:BMR ||| & 1.07 & 0.28 & $0.75^{\star \star} \dagger$ & 0.44 & $0.78^{* * *} \dagger$ & 0.31 & 1.07 & 0.30 & $0.91^{* *} \dagger$ & 0.30 & $0.83^{\star \star \star} \dagger$ & 0.27 \\
\hline Protein (E\%) ๆ 9 & 17.9 & $2 \cdot 8$ & $18 \cdot 4$ & 2.9 & $18 \cdot 3$ & $2 \cdot 8$ & $17 \cdot 3$ & 2.9 & $17 \cdot 6$ & $2 \cdot 8$ & $18 \cdot 5$ & $3 \cdot 1$ \\
\hline Fat $(E \%)$ & $32 \cdot 0$ & $5 \cdot 1$ & $32 \cdot 3$ & $4 \cdot 7$ & 33.2 & $6 \cdot 3$ & 33.4 & $5 \cdot 6$ & $31 \cdot 4^{*}$ & $5 \cdot 1$ & 32.5 & $6 \cdot 0$ \\
\hline SFA (E\%) & 14.0 & $2 \cdot 3$ & $14 \cdot 2$ & $3 \cdot 3$ & 14.5 & 3.8 & 14.2 & $2 \cdot 7$ & $13 \cdot 0^{* *} \dagger$ & $2 \cdot 3$ & $14 \cdot 1$ & $3 \cdot 2$ \\
\hline MUFA (E\%) & 10.4 & $2 \cdot 2$ & $10 \cdot 1$ & 1.7 & 10.5 & $2 \cdot 1$ & $10 \cdot 6$ & $2 \cdot 0$ & $9 \cdot 8^{\star}$ & $1 \cdot 8$ & 10.5 & $2 \cdot \overline{3}$ \\
\hline PUFA (E\%) & 5.0 & 1.6 & 5.0 & 1.9 & $5 \cdot 2$ & 1.7 & $5 \cdot 8$ & $2 \cdot 3$ & 5.4 & 1.9 & $5 \cdot 2$ & 1.8 \\
\hline Carbohydrates (E\%) & $50 \cdot 1$ & $5 \cdot 1$ & $49 \cdot 3$ & $5 \cdot 2$ & 48.5 & $6 \cdot 7$ & $49 \cdot 1$ & $6 \cdot 3$ & $51 \cdot 0$ & $5 \cdot 6$ & $49 \cdot 0$ & $6 \cdot 3$ \\
\hline Added sugar ( $\mathrm{E} \%)$ & $10 \cdot 8$ & $5 \cdot 1$ & $8 \cdot 0^{\star *} \dagger$ & $4 \cdot 3$ & 9.5 & $5 \cdot 8$ & $9 \cdot 3$ & $5 \cdot 5$ & 9.9 & $5 \cdot 6$ & 8.5 & $5 \cdot 3$ \\
\hline Fibre (g/MJ) & $2 \cdot 3$ & 0.5 & $2 \cdot 5^{\star} \dagger$ & 0.6 & $2 \cdot 3$ & 0.5 & $2 \cdot 4$ & 0.6 & 2.5 & 0.5 & $2 \cdot 4$ & 0.6 \\
\hline Protein $(\mathrm{g})$ & 75.9 & $15 \cdot 3$ & 69.5 & $13 \cdot 8$ & $71 \cdot 6$ & $15 \cdot 6$ & $75 \cdot 6$ & $18 \cdot 0$ & $67 \cdot 8^{\star \star} \dagger$ & $15 \cdot 8$ & 71.7 & $15 \cdot 6$ \\
\hline Fat $(\mathrm{g})$ & 64.6 & 23.3 & $56 \cdot 1$ & 13.8 & $60 \cdot 6$ & $16 \cdot 9$ & $68 \cdot 6$ & $21 \cdot 3$ & $57 \cdot 4^{* \star} \dagger$ & 18.6 & $59 \cdot 5^{\star \star} \dagger$ & $19 \cdot 6$ \\
\hline SFA (g) & $28 \cdot 3$ & $10 \cdot 0$ & $24 \cdot 2^{*}$ & 6.4 & $26 \cdot 4$ & $8 \cdot 8$ & 28.9 & $9 \cdot 4$ & $23 \cdot 7^{\star \star \star} \dagger$ & 8.1 & $25 \cdot 7$ & 9.1 \\
\hline MUFA (g) & $20 \cdot 7$ & 9.2 & $17 \cdot 8$ & $4 \cdot 7$ & $19 \cdot 1$ & $5 \cdot 3$ & $20 \cdot 7$ & 8.2 & $17 \cdot 8^{*}$ & $6 \cdot 1$ & $19 \cdot 1$ & 6.5 \\
\hline PUFA (g) & $10 \cdot 0$ & 4.4 & 9.2 & 4.7 & $9 \cdot 6$ & $4 \cdot 1$ & $12 \cdot 0$ & $5 \cdot 8$ & $10 \cdot 0$ & $5 \cdot 0$ & $9 \cdot 6^{\star *} \dagger$ & $5 \cdot 1$ \\
\hline Carbohydrates (g) & $207 \cdot 3$ & 39.9 & $180 \cdot 0^{*}$ & $42 \cdot 2$ & $183 \cdot 5^{\star \star} \dagger$ & $35 \cdot 7$ & $210 \cdot 8$ & 48.6 & $193 \cdot 7$ & 51.0 & $185 \cdot 9^{\star \star} \dagger$ & 48.0 \\
\hline Added sugar (g) & 47.5 & $27 \cdot 8$ & $33 \cdot 7^{\star}$ & $20 \cdot 8$ & $38 \cdot 0^{*}$ & $24 \cdot 1$ & 41.8 & 28.0 & 41.9 & 27.5 & 34.9 & 23.5 \\
\hline Fibre (g) & $16 \cdot 5$ & 3.5 & $16 \cdot 4$ & 5.4 & $15 \cdot 3$ & 3.5 & 17.4 & 4.5 & $16 \cdot 3$ & $4 \cdot 6$ & $16 \cdot 0$ & $5 \cdot 3$ \\
\hline
\end{tabular}

TLG, therapist-led group; SHG, self-help group; EI, energy intake; E\%, percentage of energy intake.

Within-group change from baseline to 6 months or baseline to 24 months calculated with paired-samples $t$ tests: ${ }^{*} P<0.05,{ }^{* \star} P<0.01,{ }^{* * *} P<0.001$

† Significant when adjusted for multiplicity ${ }^{(13)}$

There were no differences between the two groups at baseline, 6 months or 24 months calculated with independent-samples $t$ tests.

$\|$ Except for the change in E\% from added sugar from baseline to 6 months, there were no between-group differences in change from baseline to 6 and 24 months calculated with independent-samples $t$ tests.

I Body fat was measured by dual-energy $\mathrm{X}$-ray absorptiometry.

¥¥BMI$z$-score was calculated according to Cole et al ${ }^{(10)}$.

$\S \S$ El was estimated from $4 \mathrm{~d}$ food records.

III| BMR was calculated with Schofield equations ${ }^{(12)}$.

I ๆ Percentage of daily El. 
In both intervention groups, BF, BMI $z$-scores and energy intake significantly decreased from baseline to 6 months and from baseline to 24 months (Table 2; see Fig. 1 for the flow of the participants). In the TLG and SHG, respectively, a reduction in BMI $z$-scores of 0.22 and 0.19 units was observed from baseline to 6 months, and a reduction of $0 \cdot 18$ and 0.17 units was seen from baseline to 24 months. Concomitant with this, a 4.7 and $4.4 \%$ reduction in $\mathrm{BF}$ was seen from baseline to 6 months, and a 4.8 and $5 \%$ reduction in $\mathrm{BF}$ from baseline to 24 months in the TLG and SHG, respectively.

The dietary macronutrient composition changed, with mean E\% from added sugar decreasing $(P<0 \cdot 01)$ and mean $\mathrm{g}$ fibre/ MJ increasing $(P<0.05)$ from baseline to 6 months in the TLG and mean $\mathrm{E} \%$ from SFA decreasing from baseline to 6 months $(P<0.01)$ in the SHG, but these changes were not sustained after 24 months. In both groups, the reported mean $\mathrm{E} \%$ from SFA was $3.0-4.5 \%$ above Norwegian recommendations at all assessment points, whereas mean $\mathrm{E} \%$ from MUFA was $0.2 \%$ below the recommended level at 6 months in the SHG and mean E\% from added sugar was $0.8 \%$ above the recommended level at baseline in the TLG. The mean $\mathrm{E} \%$ from carbohydrates were 0.7 and $1.5 \%$ below the recommended level at 6 and 24 months, respectively, in the TLG, and 0.9 and $1.0 \%$ below the recommended level at baseline and 24 months, respectively, in the SHG. The mean $\mathrm{E} \%$ of the remaining macronutrients was in line with Norwegian recommendations. Apart from a significant decrease in energy intake/kg in both intervention groups, no significant changes were found from 6 to 24 months for BF, BMI $z$-scores or dietary intake in either group.

\section{Discussion}

To our knowledge, the present study is the first to compare the long-term changes in adiposity and dietary intake accompanying different ways to involve parents in family-based child obesity treatment. The key study findings were that no significant differences were detected for the change in adiposity and dietary intake between children of parents in the TLG and SHG. In both groups, the children achieved a significant reduction in BF and BMI $z$-scores after 6 months, which persisted throughout 24 months of treatment. Also, both groups achieved a significant reduction in energy intake from baseline to 6 months, which was sustained after 24 months, with an even further reduction in energy intake/ $\mathrm{kg}$ from 6 to 24 months in both groups. In contrast to many child obesity treatment studies included in systematic reviews ${ }^{(1,6)}$, the present study had generalisable recruitment methods, true randomisation, long-term treatment and high retention rates, and a short-term as well as a long-term assessment was conducted. Using BF measured by dual-energy X-ray absorptiometry as an outcome further strengthens the findings, as this is a more specific measure of obesity which is more accurate and sensitive to change compared with measures of only height and weight in children ${ }^{(14)}$.

In the present study, no between-group differences in the effectiveness of the interventions were found. It is therefore possible that the support received by the parents in the SHG may have been as effective as the education parents received in the TLG, or the reverse, parent groups were not the most effective part of the intervention. Similar reductions in adiposity after child obesity treatment conducted under a wide range of conditions were found in a recent meta-analysis ${ }^{(2)}$, and studies using wait-listed controls have found significant improvements in adiposity in the control condition ${ }^{(15)}$. These findings indicate that when some main components are present, interventions may be efficient in the short term, regardless of how treatment is delivered, and parent readiness to change has been suggested as one such key predictor of treatment success ${ }^{(8)}$. The detection of obesity through recruitment and baseline assessment may be sufficient, by making families aware of the child's obesity and motivating them to change behaviour ${ }^{(16)}$. It is therefore possible that the comparable improvement in children's adiposity seen in both intervention groups was a result of the parents' readiness to make lifestyle changes.

Children with parents in the TLG and SHG achieved a significant reduction in BMI $z$-scores of 0.22 and 0.19 units from baseline to 6 months, whereas others have found a 0.31 unit reduction $^{(17)}$ and a $0 \cdot 10$ unit reduction $^{(16)}$, both reported at the end of 6-month family-based interventions. Few child obesity treatment studies have reported a change in BF, but one high-intensity, family-based 12-month programme including exercise, dietary and behaviour modification has reported a $4.0 \%$ reduction in $\mathrm{BF}$ after 12 months ${ }^{(18)}$. The 4.8 and $5 \%$ reduction in $\mathrm{BF}$ after 24 months found in the TLG and SHG, respectively, of the present study is thus an interesting finding, as the SHG of the present study is estimated to be much less resource-intensive compared with the aforementioned highintensity programme. The substantial reduction in $\mathrm{BF}$ and the modest reduction in BMI $z$-score may indicate that the children acquired increased muscle mass. To our knowledge, no study comparable with the present study has been published on child obesity treatment with 24 months duration. The clinical significance of adiposity reduction found in the present study may be questioned, as one study ${ }^{(19)}$ has suggested that a reduction in BMI $z$-scores of at least 0.25 is required to improve adiposity and metabolic health in obese adolescents. A recent study, however, has found that even a modest reduction in BMI $z$-score of $<0 \cdot 1$ is associated with improvement in several cardiovascular risk factors ${ }^{(20)}$, which would make the results of the present study clinically significant.

The present study focused on gradual changes that the families were expected to be able to maintain over time, as emphasised by an expert committee on obesity treatment in children $^{(8)}$. An intervention period of 24 months should be of adequate duration to see substantial reductions in adiposity, whereas other benefits of behavioural obesity treatment (e.g. weight-related behaviours such as dietary habits) may be more noticeable in such a short term ${ }^{(8)}$. In the present study, a reduction in adiposity and energy intake persisted from 6 to 24 months of treatment, i.e. 18 months after the intensive part of the intervention in both TLG and SHG. These findings are of great interest, as they could imply that the participating families acquired important changes in 
eating habits independent of the parental intervention. In the long run, these beneficial eating habits could contribute to a reduced risk of childhood obesity tracking into adult life ${ }^{(21)}$ for the participating children, particularly if the children's muscle mass was increased, thereby increasing the proportion of metabolically active tissue and hence BMR. Changes in dietary intake in the present study are consistent with similar findings from the few child obesity interventions reporting this outcome $^{(22-24)}$, namely a decrease in energy intake, concomitant with minimal changes in macronutrient composition.

The present study has some limitations. We did not include a no-treatment control group in the present study and thus the change in BMI $z$-score may just reflect the natural course of the children's growth. However, it is reasonable to assume that both intervention groups would have been successful relative to a no-intervention control group, as findings from several studies ${ }^{(15,25,26)}$ indicate that adiposity continues to increase in obese children not receiving treatment. There are ethical issues associated with having a long-term no-intervention group in child obesity studies ${ }^{(27)}$, and some have argued that because an intervention is established to be more effective than the passage of time, there is little to be learned from repeated comparisons with no-treatment or wait-listed controls $^{(28)}$. Also, no information was available regarding the puberty stage of the children, meaning that we had an unknown ratio of pre-pubertal to pubertal children in the present study. The puberty stage may have affected the children's level of physical activity; however, we have recently published that the physical activity of these children was in fact decreased after 6 and 24 months $^{(29)}$, supporting the belief that reduced adiposity in the children in the present study was mainly due to reduced energy intake. Also, the inherent difficulties of measuring dietary intake in children are well recognised $^{(30,31)}$, being prone to reporting error, mostly through under-reporting ${ }^{(30)}$, which increases with children's age $^{(32)}$ and with increasing adiposity ${ }^{(33)}$, thus the present findings should be interpreted in the context of some limitations. It is nevertheless important to report dietary intakes in children to evaluate which eating habits are amenable to change ${ }^{(30)}$, and using the same dietary assessment method throughout the study increases the likelihood of systematic rather than random errors. The estimated food record is considered an accurate dietary assessment tool ${ }^{(34)}$, with the ability to provide an estimate of energy and macronutrient intake when reported for 3-10d, including week and weekend days. Comparing foods with photographs has been recognised as potentially improving estimates of dietary intake in a paediatric population ${ }^{(35)}$ and is less burdensome for the participants than a weighed food record ${ }^{(35)}$. Despite the limitations, we believe that the relative reduction in reported energy intake is real, based on the observed reduction in adiposity during the treatment period.

There is little doubt that cost-effectiveness will become an increasingly important consideration in future health decisions. As the two intervention groups in the present study were equally effective, a logical inference is that the least expensive intervention is the more cost-effective. The potential resource savings of the SHG, instead of the TLG, have important health cost implications. The SHG might be the solution to the scarcity of health professionals in primary health care. However, it is yet to be determined whether the SHG are equally effective as other types of lifestyle interventions. Based on these findings, we recommend that clinicians should focus on the child's energy intake and explore the possibilities for reducing the child's total energy intake together with the family, focusing on both type and the amount of food eaten.

In conclusion, the present randomised parallel-group trial demonstrated that obese children participating in two family-based interventions including TLG and SHG for parents achieve equally positive, persistent improvements in adiposity and dietary intake. Improvements in adiposity and dietary intake were sustained from 6 to 24 months of treatment, i.e. 18 months after the intensive part of the intervention. This could imply that the children acquired favourable changes in their eating habits that combined with a reduced BF mass and a possibly increased muscle mass may contribute to a reduced risk for childhood obesity tracking into adult life. Considerable work remains to determine the optimal way to involve parents to achieve persistent, cost-effective reduction in the adiposity of obese children. Further research should be performed to clarify whether the SHG should be preferred to parental group treatment for similar children with obesity.

\section{Acknowledgements}

We thank all children and parents who have contributed to the present study. We are also grateful to our colleagues at St Olav University Hospital. The present study was supported by the Liaison Committee for Central Norway Regional Health Authority; the National Council of Mental Health/Health and Rehabilitation, NTNU; St Olav University Hospital; the Bergen Medical Research Foundation and the Meltzer Foundation. S. S., R. $\varnothing$. and L. W. contributed to the study design. S. S. and R. $\varnothing$. contributed to the conduction of the study. All authors contributed to the analysis and interpretation of the data. H. T. H., S. S., L. W. and O. A. G. performed the statistical analysis. H. T. H. drafted the manuscript. All authors critically revised the final manuscript. The authors declare no conflicts of interest.

\section{References}

1. Oude Luttikhuis H, Baur L, Jansen H, et al. (2009) Interventions for treating obesity in children. The Cochrane Database of Systematic Reviews, issue 1, CD001872.

2. Kitzmann KM, Dalton WT III, Stanley CM, et al. (2010) Lifestyle interventions for youth who are overweight: a meta-analytic review. Health Psychol 29, 91-101.

3. Epstein LH, Paluch RA, Roemmich JN, et al. (2007) Familybased obesity treatment, then and now: twenty-five years of pediatric obesity treatment. Health Psychol 26, 381-391.

4. Golan M, Kaufman V \& Shahar DR (2006) Childhood obesity treatment: targeting parents exclusively $v$. parents and children. Br J Nutr 95, 1008-1015.

5. Reinehr T (2011) Child-centred physical activity programme and parent-centred dietary programme alone or combined 
lead to sustainable reductions in BMI in 5-10-year-olds: diet alone or diet plus activity programmes seemed most effective. Evid Based Nurs 14, 12-13.

6. Collins CE, Warren J, Neve M, et al. (2006) Measuring effectiveness of dietetic interventions in child obesity: a systematic review of randomized trials. Arch Pediatr Adolesc Med 160, 906-922.

7. Collins CE, Okely AD, Morgan PJ, et al. (2011) Parent diet modification, child activity, or both in obese children: an RCT. Pediatrics 127, 619-627.

8. Barlow SE (2007) Expert committee recommendations regarding the prevention, assessment, and treatment of child and adolescent overweight and obesity: summary report. Pediatrics 120, Suppl. 4, S164-S192.

9. Norwegian Directory of Health (2005) Norwegian Recommendations for Nutrition and Physical Activity, vol. 2011. Oslo: Norwegian Directory of Health.

10. Cole TJ, Bellizzi MC, Flegal KM, et al. (2000) Establishing a standard definition for child overweight and obesity worldwide: international survey. BMJ 320, 1240-1243.

11. Lillegaard IT, Overby NC \& Andersen LF (2005) Can children and adolescents use photographs of food to estimate portion sizes? Eur J Clin Nutr 59, 611-617.

12. Schofield WN (1985) Predicting basal metabolic rate, new standards and review of previous work. Hum Nutr Clin Nutr 39, Suppl. 1, 5-41.

13. Benjamini Y \& Hochberg Y (1995) Controlling the false discovery rate: a practical and powerful approach to multiple testing. J R Stat Soc 57, 289-300.

14. Helba M \& Binkovitz LA (2009) Pediatric body composition analysis with dual-energy X-ray absorptiometry. Pediatr Radiol 39, 647-656.

15. Golley RK, Magarey AM, Baur LA, et al. (2007) Twelvemonth effectiveness of a parent-led, family-focused weightmanagement program for prepubertal children: a randomized, controlled trial. Pediatrics 119, 517-525.

16. Hughes AR, Stewart L, Chapple J, et al. (2008) Randomized, controlled trial of a best-practice individualized behavioral program for treatment of childhood overweight: Scottish Childhood Overweight Treatment Trial (SCOTT). Pediatrics 121, e539-e546.

17. Okely AD, Collins CE, Morgan PJ, et al. (2010) Multi-site randomized controlled trial of a child-centered physical activity program, a parent-centered dietary-modification program, or both in overweight children: the HIKCUPS study. J Pediatr 157, 388-394, 394.e1.

18. Savoye M, Shaw M, Dziura J, et al. (2007) Effects of a weight management program on body composition and metabolic parameters in overweight children: a randomized controlled trial. JAMA 297, 2697-2704.

19. Ford AL, Hunt LP, Cooper A, et al. (2010) What reduction in BMI SDS is required in obese adolescents to improve body composition and cardiometabolic health? Arch Dis Child 95, 256-261.
20. Kolsgaard ML, Joner G, Brunborg C, et al. (2011) Reduction in BMI $z$-score and improvement in cardiometabolic risk factors in obese children and adolescents. The Oslo Adiposity Intervention Study - a hospital/public health nurse combined treatment. BMC Pediatr 11, 47.

21. Reilly JJ, Methven E, McDowell ZC, et al. (2003) Health consequences of obesity. Arch Dis Child 88, 748-752.

22. Alexy U, Reinehr T, Sichert-Hellert W, et al. (2006) Positive changes of dietary habits after an outpatient training program for overweight children. Nutr Res 26, 202-208.

23. Burrows T, Warren JM, Baur LA, et al. (2008) Impact of a child obesity intervention on dietary intake and behaviors. Int J Obes (Lond) 32, 1481-1488.

24. Waling M, Lind T, Hernell O, et al. (2010) A one-year intervention has modest effects on energy and macronutrient intakes of overweight and obese Swedish children. $J$ Nutr 140, 1793-1798.

25. Wright CM, Emmett PM, Ness AR, et al. (2010) Tracking of obesity and body fatness through mid-childhood. Arch Dis Child 95, 612-617.

26. Wilfley DE, Tibbs TL, Van Buren DJ, et al. (2007) Lifestyle interventions in the treatment of childhood overweight: a meta-analytic review of randomized controlled trials. Health Psychol 26, 521-532.

27. Warren JM, Golley RK, Collins CE, et al. (2007) Randomised controlled trials in overweight children: practicalities and realities. Int J Pediatr Obes 2, 73-85.

28. Gilles A, Cassano M, Shepherd EJ, et al. (2008) Comparing active pediatric obesity treatments using meta-analysis. J Clin Child Adolesc Psychol 37, 886-892.

29. Steinsbekk S, Wichstrom L, Odegard R, et al. (2012) Change in body fat during a family-based treatment of obesity in children: the relative importance of energy intake and physical activity. Obes Facts 5, 515-526.

30. Collins CE, Watson J \& Burrows T (2010) Measuring dietary intake in children and adolescents in the context of overweight and obesity. Int J Obes (Lond) 34, 1103-1115.

31. Magarey A, Watson J, Golley RK, et al. (2010) Assessing dietary intake in children and adolescents: considerations and recommendations for obesity research. Int $\mathrm{J}$ Pediatr Obes 6, 2-11.

32. Bandini LG, Must A, Cyr H, et al. (2003) Longitudinal changes in the accuracy of reported energy intake in girls $10-15$ y of age. Am J Clin Nutr 78, 480-484.

33. Fisher JO, Johnson RK, Lindquist C, et al. (2000) Influence of body composition on the accuracy of reported energy intake in children. Obes Res 8, 597-603.

34. Biro G, Hulshof KF, Ovesen L, et al. (2002) Selection of methodology to assess food intake. Eur J Clin Nutr 56, Suppl. 2, S25-S32.

35. Higgins JA, LaSalle AL, Zhaoxing P, et al. (2009) Validation of photographic food records in children: are pictures really worth a thousand words? Eur J Clin Nutr 63, 1025-1033. 